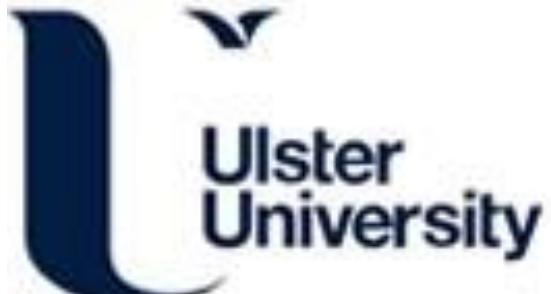

\section{Detection of Workplace Sedentary Behavior using Thermal Sensors}

Synnott, J., Rafferty, J., \& Nugent, C. (2016). Detection of Workplace Sedentary Behavior using Thermal Sensors. In Unknown Host Publication (pp. 5413-5416). IEEE. https://doi.org/10.1109/EMBC.2016.7591951

Link to publication record in Ulster University Research Portal

\author{
Published in: \\ Unknown Host Publication
}

Publication Status:

Published online: 18/10/2016

DOI:

10.1109/EMBC.2016.7591951

\section{Document Version}

Publisher's PDF, also known as Version of record

\section{General rights}

Copyright for the publications made accessible via Ulster University's Research Portal is retained by the author(s) and / or other copyright owners and it is a condition of accessing these publications that users recognise and abide by the legal requirements associated with these rights.

\section{Take down policy}

The Research Portal is Ulster University's institutional repository that provides access to Ulster's research outputs. Every effort has been made to ensure that content in the Research Portal does not infringe any person's rights, or applicable UK laws. If you discover content in the Research Portal that you believe breaches copyright or violates any law, please contact pure-support@ulster.ac.uk. 


\title{
Detection of Workplace Sedentary Behavior using Thermal Sensors
}

\author{
J. Synnott, J. Rafferty, and C. D. Nugent, Member, IEEE
}

\begin{abstract}
Sedentary behavior has been linked to leading causes of morbidity and mortality, including cancer, cardiovascular disease and diabetes. Those who work in office workplaces are susceptible to higher levels of sedentary behavior during the working day. This paper introduces a novel approach to the detection of sedentary behavior through the use of a thermal sensor mounted on the ceiling above a busy workspace. This solution was found to more accurately record 7 out of 10 activity metrics in comparison to self-assessment, when compared to chair pressure sensor recordings.
\end{abstract}

\section{INTRODUCTION}

There is a growing interest in the long-term assessment of physical activity (PA) levels in the healthy population. Participation in low levels of PA or high levels of sedentary behavior (SB) may result in increased prevalence of chronic conditions. The World Health Organization has provided global recommendations for the PA level needed for the prevention of noncommunicable diseases, depression and cognitive decline. For those aged 18 and above, it is recommended that at least 150 minutes of moderate-intensity or 75 minutes of vigorous-intensity aerobic PA are performed per week [1]. The intensity level of an activity can be expressed as Metabolic Equivalent of Task (MET), which is defined as a ratio of metabolic rate during a PA to the metabolic rate of the average person seated at rest. Moderateintensity physical activities have an energy expenditure of approximately 3-6 METs and include activities such as housework and domestic chores, brisk walking and gardening. Vigorous-intensity physical activities have energy expenditure greater than 6 METs, and include activities such as running, hill walking and fast swimming [2].

SB is defined as waking behavior with an energy expenditure less than or equal to 1.5 metabolic METs performed while in a sitting or reclining posture [3]. SBs include many activities that are common in an office workplace, including using a computer, reading, and sitting during discussions [3,4]. Many desk-based occupations typically have METs which barely exceed the threshold to be classed as SB. Examples include: Management (1.73 METs), Computer \& Mathematical (1.58 METs), Legal (1.50 METs), and Office \& Administrative Support (1.83 METs). A full summary of MET values can be found on the National Cancer Institute's website [5].

Studies have shown that older adults engage in increased amounts of SB. For example, a study involving 6,329

Invest Northern Ireland is acknowledged for supporting this project under the Competence Centre Programme Grant RD0513853 - Connected Health Innovation Centre. Additional support provided by the Department of Employment and Learning, Northern Ireland.

J. Synnott, J. Rafferty and C.D. Nugent are with the Computer Science Research Institute and the School of Computing and Mathematics, University of Ulster (phone: +44 2890368840 e-mail: \{j.synnott, j.rafferty, cd.nugent\}@ulster.ac.uk). participants found that the amount of daily SB time increased in age groups above the age of 40 . The mean hours per day of SB was 7.55 for those aged 40-49, 7.87 for those aged 50$59,8.41$ for those aged $60-69$ and 9.28 for those aged $70-85$ [6]. The health benefits of engaging in the recommended amounts of moderate to vigorous PA (MVPA) are well documented in literature. It is possible, however, to engage in the recommended level of MVPA while still engaging in high levels of SB $[3,4]$. Emerging evidence indicates that SB may have an independent role on health, and it may still be possible to experience the risk factors associated with SB whilst achieving MVPA goals. Such risk factors include chronic, noncommunicable diseases such as cardiovascular disease, diabetes and cancer [3,4]. As a result, it has been suggested that there may need to be an adjustment in the prescription of the optimal daily human activity patterns for health [7].

There has previously been a lack of research into the direct monitoring of SB levels, and it has been suggested that the monitoring of SB levels should receive as much attention as the monitoring of MVPA levels [8]. SB monitoring has therefore become a new focus for research on PA and health. It is understood that through the long term, frequent monitoring of SB levels, it is possible to identify inadequate levels and invoke change before negative effects occur. Given the low typical MET values of many desk-based occupations, the office workplace serves as a suitable target for intervention. The result may be a healthier and more productive workplace.

This paper introduces a novel approach for the monitoring of SB in the workplace. This approach incorporates the use of a thermal sensor mounted on the ceiling to detect the presence and absence of workers at a work desk. The approach is capable of determining 10 metrics detailing desk occupancy, including average occupancy time, average absence time, and the number of desk vacancies throughout the day. Section 2 provides an overview of related work. Section 3 provides details on the proposed approach. Section 4 describes the implementation of the approach. Section 5 details the results from the testing of the approach and provides an evaluation of effectiveness. Finally, Section 6 provides concluding remarks and suggestions for future work.

\section{RELATED WORK}

Gardner et al. [9] have completed a review of behavior change strategies used to reduce SB among adults. They evaluated 38 interventions, of which 20 were based in the workplace. $21 \%$ of these studies evaluated worksite sitting time only, and $7 \%$ evaluated both waking and worksite sedentary time. $73 \%$ of the evaluated studies relied upon selfreporting as a measure of $\mathrm{SB}$, and $27 \%$ of studies used accelerometers as objective measures. The most promising 
interventions specifically targeted SB rather than PA. Additionally, the most promising interventions focused on environmental restructuring, persuasion or education.

Atkin et al. [10] highlighted the importance of high quality measurement of SB for determining associations with health outcomes and for the development and evaluation of behavior change interventions. They provided an overview of subjective and objective measurements of SB which are suitable for use with both children and adults. They highlighted that existing subjective approaches to SB monitoring, such as questionnaires, focus on screen-based activities such as television watching. They noted that such subjective measures are moderately reliable but have slight to moderate validity. The increased use of accelerometers for SB monitoring has been noted, and it is stated that these solutions offer improvements over such self-reporting measures.

Rosenberger et al. [11] described the use of hip and wristworn accelerometers for the estimation of activity and SB, noting that hip-worn accelerometers were able to provide a more accurate prediction of energy expenditure associated with activity, compared to wrist-worn accelerometers. Hart et al. [12] investigated the number of days of monitoring required to reliably predict $\mathrm{PA}$ and $\mathrm{SB}$ in older adults across 3 sources: pedometer, accelerometer and self-assessed PA $\log$. They incorporated the use of an ActiGraph 7164 accelerometer for the capture of SB, which required 5 days of complete data to reliably predict total SB time.

The proposed approach in this paper aims to expand upon lessons learned from existing work by offering an objective, unobtrusive approach to SB monitoring in the workplace.

\section{PROPOSED APPROACH}

The proposed approach focusses on the use of an unobtrusive environment-based sensor deployed on the ceiling of an office for the long-term tracking of SB in the workplace. In particular, this approach incorporates the use of a thermal sensor capable of detecting the presence and movement of heat sources within a scene.

A low resolution, low cost thermal sensor deployed on the ceiling was selected for several reasons. Firstly, such an approach facilitates unobtrusive, always-on monitoring. In contrast to wearable devices, this approach does not require users to remember to wear a device in a specific location, or charge a device. Additionally, such thermal sensors often have fields of view which are capable of monitoring the movements of multiple workspaces simultaneously in shared work environments, reducing the number of devices required. Thermal sensing technology is also capable of monitoring movement regardless of fluctuation in light levels. Finally, such sensors provide advantages over chair pressure sensors by allowing users to move freely in movable office chairs without restrictions by cables, and can operate with both sitting and standing desks.

\section{IMPLEMENTATION}

The chosen thermal sensor was an Omron D6T-1616L, which has a resolution of 16x16, a 90-degree field of view providing a coverage area of $6 \mathrm{~m}$ by $6 \mathrm{~m}$ at a height of $3 \mathrm{~m}$, and a sample rate of $3.5 \mathrm{~Hz}$. The sensor was mounted using a portable, height adjustable frame with the sensor facing downwards, providing a birds-eye view of the recording area. This was chosen to minimize occlusions and to represent a long term deployment in which the sensor would be attached to the ceiling. The sensor produces a serial stream of thermal data which is transmitted via USB to a listener. This listener verifies the structure of the data before transmitting to a central server for storage in a MySQL database. The server then processes the raw data to identify the presence of heat sources. Heat source identification is performed through foreground detection and blob tracking algorithms implemented using MATLAB.

The foreground detection algorithm was designed to facilitate the detection of moving objects in addition to a predominantly stationary heat source, whilst minimizing the impact of fluctuating heat sources such as environmental temperature drift and warm objects such as hot drinks or computing equipment. The approach achieves this by first capturing a background thermal reading of a scene only containing stationary objects, such as desks and computing equipment. A binary mask is then generated for each subsequent frame of thermal data, in which any values which deviate from the background snapshot by more than a set threshold are assigned a logical 1 value. All other pixels are assigned a logical 0 value. This binary mask is then analyzed using MATLAB's BlobAnalysis class in order to determine the area, bounding box, centroid and eccentricity of any heat sources with an area greater than 8 pixels. This was determined to give the most consistent human tracking performance while filtering out other heat sources such as smaller computing equipment and warm drinks. Such qualifying heat sources are referred to as 'blobs' in the remainder of this paper.

A significant source of temperature variation not due to human activity is environmental temperature drift. This is due to significant increases in office temperature throughout the day due to sunlight, computing equipment and room occupants. In order to minimize the impact of temperature drift, the foreground detection algorithm was designed to update the background reference reading when no blobs were detected. As a result, the foreground detection algorithm would automatically re-calibrate when no inhabitants were within the field of view. To minimize the impact of temperature drift while inhabitants were within the field of view of the sensor, the foreground detection algorithm was also designed to track the temperature variation over time of each pixel. The algorithm was designed to sample every pixel every 60 seconds. Pixels that were determined to have a steady increase in temperature over the previous 5 samples were identified as objects heating up, rather than room inhabitants. When such a situation was detected, the background value of the affected pixel would be updated to the current temperature value of that pixel.

As such environments are typically shared by multiple occupants it is necessary to track and individually analyze the movement of separate heat sources for the calculation of individual metrics. This was achieved by assigning blobs to individual tracks by comparing the current frame of data with the previous frame, and calculating the Euclidean distance between the past and current centroids of each blob. Each blob is then assigned to the track of the blob with the smallest 
Euclidean distance. The tracking of human heat sources within a scene facilitated the calculation of a series of 17 metrics. These metrics were designed to facilitate the analysis of areas including: movement amount, occupant and ambient temperature, occupant count, occupant proximity and time without occupancy. Rafferty et al. [13] provide a detailed overview of these metrics and the associated architecture. For the purposes of SB analysis, this paper focusses on the use of 10 metrics describing occupancy and movement amount. These are: Time in Workplace, Total Occupancy Time, Total Absence Time, Median/Mean Occupancy Time, Median/Mean Absence Time, Number of Absences, Maximum Occupancy Time, and Maximum Absence Time.

The sensor was positioned in an office above a researcher's work desk, ensuring the field of view covered the entire desk and the researcher's chair when occupied. Figure 1 illustrates the deployment setup.

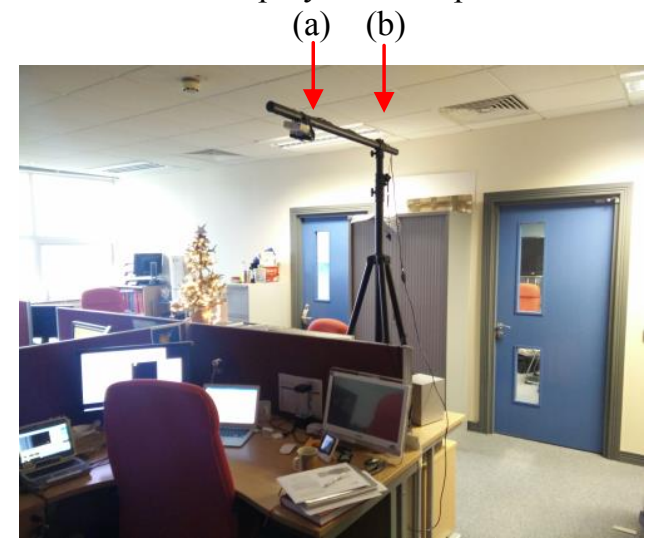

Figure 1. Deployment of the thermal sensor (a). A frame (b) was used to mount the sensor in order to facilitate mobility for testing.

This particular environment was chosen as it was representative of a typical office environment in which there would be a variety of sources of noise. Such noise includes sources of heat not relating to that of the researcher occupying the work desk. For example, other researchers moving within the field of view of the sensor, office equipment including monitors and printers heating up, warm drinks, and residual heat emanating from previously occupied chairs. Additionally, the temperature was not controlled in the environment, resulting in significant fluctuations in temperature throughout the day. Such sources of noise were chosen to test the robustness of the data processing strategies developed.

Figure 2 provides an example of the thermal data converted to a grayscale image (left), and the corresponding binary mask (right). In the grayscale image, warmer areas are lighter and colder areas are darker. This particular scene contains several heat sources including (a) a cup of coffee, (b) a participant, (c) a small laptop, (d) a monitor, (e) a touch screen computer, (f) a larger laptop, and (g) a lamp. This example illustrates how the foreground detection algorithm has the ability to identify and isolate the participant, as indicated by a single blob in the binary mask. (a) (b) (c) (d)

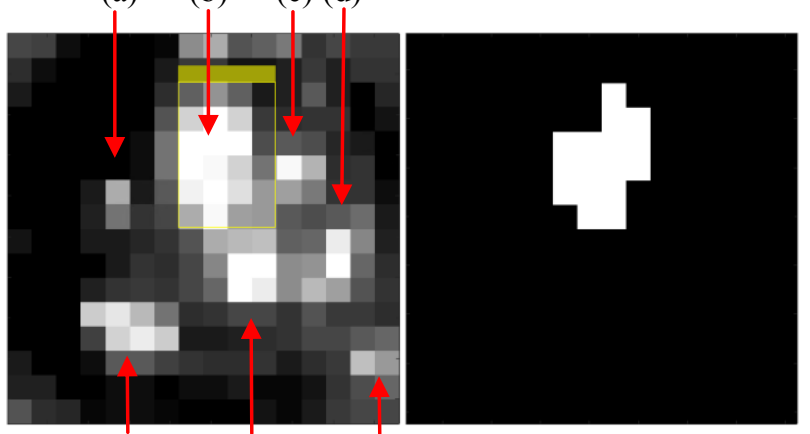

(e)

(f)

(g)

Figure 2. An example of the raw thermal data visualised as a grayscale image (left) and binary mask (right) resulting from foreground detection. Heat sources include: (a) a cup of coffee, (b) the participant, (c) a small laptop (d) a monitor, (e) a touch screen computer (f) a larger laptop, and (g) a lamp.

\section{Testing \& Evaluation}

The sensor was deployed within the environment for 3 days, recording the typical movement behaviors of one participant. The researcher was instructed not to make any intentional adjustments to daily routine or behavior in order to collect a realistic dataset. At the end of each day, the researcher was also asked to estimate metrics including the number of minutes away from their desk, and the number of breaks taken from their desk. A Tynetec Chair pressure sensor (model number: ZCS859), located on the researcher's chair, was used to provide ground truth values. Table 1 provides a comparison between the metrics calculated from the thermal sensor and the chair pressure sensor data, in addition to the self-assessment values estimated by the participant. Also presented are the mean differences in values obtained from the thermal sensor and self-assessment compared to the pressure pad, as a means of indicating accuracy.

Time in the workplace refers to the time between first desk arrival and last desk leave. Total occupancy time refers to the total cumulative time the occupant was detected throughout the day. Total Absence Time refers to the total cumulative time the occupant was not detected throughout the day. The median and mean occupancy and absence times represent the median and mean values per period of occupancy and absence detected. The number of absences represents the number of times the occupant was identified as leaving the scene. The maximum occupancy and absence times represent the longest duration of occupancy and absence periods throughout the day.

The metrics obtained from the thermal sensor were found to more closely resemble those obtained from the pressure sensor baseline for 7 out of 10 metrics. These metrics were: Total Occupancy Time, Total Absence Time, Median \& Mean Occupancy Time, Median \& Mean Absence Time, and Number of Absences. The thermal sensor consistently provided significantly higher results for Maximum Occupancy Time in comparison with the pressure sensor and self-assessments. This can be explained by considering the Number of Absences metric, in which the thermal sensor, in most cases, provided considerably lower readings than the pressure 
TABLE I. RESULTS COLLECTED FROM ANALYSIS OF THERMAL SENSOR, PRESSURE SENSOR, AND SELF-ASSESSMENT (SA) DATA. TEMPORAL DATA IS PROVIDED IN THE FORMAT: H:MM:SS. MEAN \% DIFFERENCE FROM PRESSURE SENSOR VALUES IS ALSO PROVIDED.

\begin{tabular}{|c|c|c|c|c|c|}
\hline \multirow[b]{2}{*}{ Metric } & \multirow[b]{2}{*}{ Source } & \multicolumn{3}{|c|}{ Day } & \multirow{2}{*}{\begin{tabular}{|c|} 
Mean \\
diff. \\
from \\
Pressure
\end{tabular}} \\
\hline & & 1 & 2 & 3 & \\
\hline \multirow{3}{*}{$\begin{array}{c}\text { Time in } \\
\text { Workplace }\end{array}$} & Thermal & 08:59:00 & 8:30:00 & 11:05:00 & $0: 11: 56$ \\
\hline & Pressure & $9: 00: 22$ & $8: 30: 11$ & 10:30:46 & - \\
\hline & $S A$ & 9:15:00 & $8: 30: 00$ & 10:35:00 & 0:06:21 \\
\hline \multirow{3}{*}{$\begin{array}{c}\text { Total } \\
\text { Occupancy } \\
\text { Time }\end{array}$} & Thermal & $7: 16: 00$ & 3:21:00 & 9:43:00 & $0: 19: 57$ \\
\hline & Pressure & $6: 26: 00$ & $3: 12: 22$ & 9:41:48 & - \\
\hline & $S A$ & 7:00:00 & $3: 30: 00$ & 8:00:00 & 0:51:09 \\
\hline \multirow{3}{*}{$\begin{array}{c}\text { Total } \\
\text { Absence } \\
\text { Time }\end{array}$} & Thermal & 1:43:00 & 5:09:00 & 1:22:00 & 0:31:04 \\
\hline & Pressure & $2: 34: 22$ & $5: 17: 49$ & 0:48:58 & - \\
\hline & $S A$ & $2: 15: 00$ & 5:00:00 & 2:00:00 & $0: 36: 04$ \\
\hline \multirow{3}{*}{$\begin{array}{c}\text { Median/ } \\
\text { Mean } \\
\text { Occupancy } \\
\text { Time }\end{array}$} & Thermal & $\begin{array}{c}0: 09: 00 / \\
0: 22: 56\end{array}$ & $\begin{array}{c}0: 01: 00 / \\
0: 11: 49 \\
\end{array}$ & $\begin{array}{c}0: 18: 00 / \\
0: 44: 50 \\
\end{array}$ & $\begin{array}{c}0: 05: 40 / \\
0: 12: 09\end{array}$ \\
\hline & Pressure & $\begin{array}{c}0: 08: 38 / \\
0: 11: 41\end{array}$ & $\begin{array}{c}0: 14: 09 / \\
0: 17: 29\end{array}$ & $\begin{array}{c}0: 14: 31 / \\
0: 25: 17\end{array}$ & - \\
\hline & $S A$ & 1:00:00 & 0:40:00 & 1:00:00 & $\begin{array}{c}0: 40: 54 / \\
0: 35: 11\end{array}$ \\
\hline \multirow{3}{*}{$\begin{array}{c}\text { Median/ } \\
\text { Mean } \\
\text { Absence } \\
\text { Time }\end{array}$} & Thermal & $\begin{array}{c}0: 03: 30 / \\
0: 04: 46\end{array}$ & $\begin{array}{c}0: 09: 00 / \\
0: 18: 22\end{array}$ & $\begin{array}{c}0: 03: 30 / \\
0: 05: 55\end{array}$ & $\begin{array}{c}0: 03: 27 / \\
0: 05: 43\end{array}$ \\
\hline & Pressure & $\begin{array}{c}0: 0: 35 / \\
0: 04: 49\end{array}$ & $\begin{array}{c}0: 04: 06 / \\
0: 31: 46\end{array}$ & $\begin{array}{c}0: 00: 58 / \\
0: 02: 13\end{array}$ & - \\
\hline & $S A$ & $0: 15: 00$ & 1:00:00 & $0: 15: 00$ & $\begin{array}{l}0: 28: 07 / \\
0: 17: 04 \\
\end{array}$ \\
\hline \multirow{3}{*}{$\begin{array}{c}\text { \# of } \\
\text { Absences }\end{array}$} & Thermal & 19 & 17 & 13 & 10 \\
\hline & Pressure & 33 & 11 & 23 & - \\
\hline & $S A$ & 6 & 5 & 5 & 17 \\
\hline \multirow{3}{*}{$\begin{array}{c}\text { Max. } \\
\text { Occupancy } \\
\text { Time }\end{array}$} & Thermal & 1:10:00 & 1:08:00 & 2:39:00 & $0: 37: 30$ \\
\hline & Pressure & $0: 52: 39$ & $0: 38: 58$ & $1: 32: 53$ & - \\
\hline & $S A$ & 1:00:00 & 00:45:00 & 1:30:00 & 0:05:25 \\
\hline \multirow{3}{*}{$\begin{array}{c}\text { Max. } \\
\text { Absence } \\
\text { Time }\end{array}$} & Thermal & $0: 17: 00$ & 1:13:00 & 0:21:00 & $0: 41: 36$ \\
\hline & Pressure & 1:29:39 & $1: 54: 35$ & $0: 10: 27$ & - \\
\hline & $S A$ & $0: 30: 00$ & $2: 00: 00$ & $0: 45: 00$ & $0: 33: 12$ \\
\hline
\end{tabular}

sensor. This is due to the researcher getting up from their chair to retrieve objects or converse with colleagues, without leaving the field of view of the thermal sensor. Selfassessments provided a slightly more accurate assessment of Time in Workplace, however, the thermal sensor resulted in an acceptable $1.91 \%$ mean error. The self-assessments consistently under-estimated the number of absences throughout each day and consistently over-estimated the median and mean occupancy and absence times. This suggests that this particular participant left their desk more often than recalled, however, for less time than recalled.

Several issues with the study approach were noted throughout. In particular, the heat drift compensation algorithm occasionally filtered out some elements of the participant's heat source as a result of gradual increases in emissive temperature. Additionally, the wide sensor field of view occasionally resulted in co-workers' activity being logged by the sensor. Several issues were reported with the pressure sensor. These included discomfort with long-term use, the obtrusive nature of the pressure sensor, pressure pad cables getting caught on the legs of chair upon movement, and issues with inhabitant detection prior to data collection due to the pressure sensor sensitivity requiring specific calibration for the specific participant and furniture.

\section{CONCLUSION}

Low levels of MVPA and high levels of SB have been associated with various causes of morbidity and mortality. Past research has focused upon monitoring and increasing levels of MVPA, however, the monitoring and reduction of SB has received less focus. This paper has presented a novel approach to SB monitoring in the workplace through the use of a thermal sensor. A robust data analysis solution has been developed to provide accurate recordings in a real working environment. The approach has been shown to be capable of providing 10 metrics describing workplace activity. Results obtained highlight that the thermal sensor was able to more accurately determine Total Occupancy Time, Total Absence Time, Median \& Mean Occupancy Time, Median \& Mean Absence Time and Number of Absences than selfassessment, when compared to pressure sensor readings. It is envisioned that this approach could be incorporated as part of a larger intervention strategy, in which higher levels of SB would be detected and then discouraged through appropriate means. Future work will focus on a larger scale evaluation of both single and multiple occupancy tracking.

\section{REFERENCES}

[1] World Health Organization. Global Recommendations on Physical Activity for Health; WHO: Gevena, Switzerland, 2010.

[2] World Health Organization. (2016). What Is Moderate-Intensity and Vigorous-Intensity Physical Activity? http://www.who.int/dietphysicalactivity/physical_activity_intensity/en

[3] Sedentary Behaviour Research Network Letter to the Editor: Standardized Use of the Terms "Sedentary" and "Sedentary Behaviours". Appl. Physiol. Nutr. Metab. 2012, 37, 540-542.

[4] British Heart Foundation. (2012). Sedentary Behaviour Evidence Briefing. http://www.bhfactive.org.uk/files/525/sedentary_evidence_briefing.pd $\mathrm{f}$

[5] National Cancer Institute. (2016). Metabolic Equivalent (MET) Values for Activities in American Time Use Survey (ATUS) http://appliedresearch.cancer.gov/atus-met/

[6] C. Matthews, K. Chen, P. Freedson, M. Buchowski, B. Beech, R. Pate and R. Troiano, "Amount of Time Spent in Sedentary Behaviors in the United States, 2003-2004", American Journal of Epidemiology, vol. 167, no. 7, pp. 875-881, 2008.

[7] P. Katzmarzyk, "Physical Activity, Sedentary Behavior, and Health: Paradigm Paralysis or Paradigm Shift?", Diabetes, vol. 59, no. 11, pp. 2717-2725, 2010.

[8] N. Owen, G. Healy, C. Matthews and D. Dunstan, "Too Much Sitting: The population health science of sedentary behavior", Exercise and Sport Sciences Reviews, vol. 38, no. 3, pp. 105-113, 2010.

[9] B. Gardner, L. Smith, F. Lorencatto, M. Hamer and S. Biddle "How to reduce sitting time? A review of behaviour change strategies used in sedentary behaviour reduction interventions among adults.",2016. Available: http://www.ncbi.nlm.nih.gov/pubmed/26315814.

[10] A. Atkin, T. Gorely, S. Clemes, T. Yates, C. Edwardson, S. Brage, J. Salmon, S. Marshall and S. Biddle, "Methods of Measurement in epidemiology: Sedentary Behaviour", International Journal of Epidemiology, vol. 41, no. 5, pp. 1460-1471, 2012.

[11] M. Rosenberger, W. Haskell, F. Albinali, S. Mota, J. Nawyn and S. Intille, "Estimating Activity and Sedentary Behavior from an Accelerometer on the Hip or Wrist", Medicine \& Science in Sports \& Exercise, vol. 45, no. 5, pp. 964-975, 2013.

[12] T. Hart, A. Swartz, S. Cashin and S. Strath, "How many days of monitoring predict physical activity and sedentary behaviour in older adults?", Int J Behav Nutr Phys Act, vol. 8, no. 1, p. 62, 2011.

[13] J.Rafferty, J. Synnott and C.D. Nugent "A hybrid rule and machine learning based generic alerting platform for smart environments", 2016 Annual International Conference of the IEEE Engineering in Medicine and Biology Society, 2016, submitted. 